Journal of Patient-Centered

Volume 4

Issue 4 -- Health Disparities and Inequities: Part

Article 6 I

$11-6-2017$

\title{
Measuring the Impact of Patient-Engaged Research: How a Methods Workshop Identified Critical Outcomes of Research Engagement
}

\author{
Ellis C. Dillon \\ Leah Tuzzio \\ Sarah Madrid \\ Heather Olden \\ Robert T. Greenlee
}

Follow this and additional works at: https://aah.org/jpcrr

Part of the Health Services Research Commons, Other Medicine and Health Sciences Commons, and the Public Health Education and Promotion Commons

\section{Recommended Citation}

Dillon EC, Tuzzio L, Madrid S, Olden H, Greenlee RT. Measuring the impact of patient-engaged research: how a methods workshop identified Critical Outcomes of Research Engagement. J Patient Cent Res Rev. 2017;4:237-46. doi: 10.17294/2330-0698.1458

Published quarterly by Midwest-based health system Advocate Aurora Health and indexed in PubMed Central, the Journal of Patient-Centered Research and Reviews (JPCRR) is an open access, peer-reviewed medical journal focused on disseminating scholarly works devoted to improving patient-centered care practices, health outcomes, and the patient experience. 


\title{
Measuring the Impact of Patient-Engaged Research: How a Methods Workshop Identified Critical Outcomes of Research Engagement
}

\author{
Ellis C. Dillon, PhD, ${ }^{1}$ Leah Tuzzio, MPH, ${ }^{2}$ Sarah Madrid, MA,${ }^{3}$ Heather Olden, $\mathrm{MPH},{ }^{4}$ Robert T. \\ Greenlee, $\mathrm{PhD}, \mathrm{MPH}^{5}$ \\ ${ }^{1}$ Palo Alto Medical Foundation Research Institute, Palo Alto, CA; ${ }^{2}$ Kaiser Permanente Washington Health Research \\ Institute, Seattle, WA; ${ }^{3}$ Institute for Health Research, Kaiser Permanente Colorado, Denver, CO; ${ }^{4}$ Department of Public \\ Health Sciences, Henry Ford Health System, Detroit, MI; ${ }^{5}$ Marshfield Clinic Research Institute, Marshfield, WI
}
Purpose While strategies to evaluate the influence of engaging patient partners in research, such as the Patient- Centered Outcomes Research Institute (PCORI) WE-ENACT surveys, are beginning to emerge, a systematic set of measures for assessing the impact of patient engagement in research (PER) on study approaches and outcomes is lacking. This article describes a workshop and process used to identify and develop Critical Outcomes of Research Engagement (COREs). It proposes preliminary measures for assessing the impact of PER on the research process and outcomes of research studies.

Methods A group of 24 researchers and 5 patient partners participated in a PCORI-funded workshop designed to identify key research outcomes and corresponding measures to evaluate the impact of patientengaged research on those outcomes. Interactive group discussion and synthesis by workshop attendees led to a proposed set of core components of patient-engaged research by each stage of a research study as well as some overarching principles. Postworkshop discussions further distilled the output and considered potential gaps.

Results CORE components identified were: patient-centered, meaningful, team collaboration, understandable, rigorous, adaptable/integrity, legitimate, feasible, ethical and transparent, timely, and sustainable. Existing measures skew more toward measuring the process of engagement and less toward measuring downstream outcomes of patient-partner engagement in all phases of research.

Conclusions Next steps include finalizing measures, pilot testing them with the workshop participants, and building a larger community of practice to further advance this work. The new community plans to create a measurement tool and conduct a study to validate the measures. (J Patient Cent Res Rev. 2017;4:237-246.)

Keywords patient engagement, research, methods, measurement

$\mathrm{P}$ atient engagement in research (PER) is a moral imperative to many and increasingly a funder requirement - as in the case of the PatientCentered Outcomes Research Institute (PCORI), which has funded 570 projects and awarded \$1.61

Correspondence: Ellis C. Dillon, PhD,

Palo Alto Medical Foundation, 2350 W. El Camino Real, 4th floor, Mountain View, CA, 94040, T: +1-650-625-3876,

Email: dillone@pamfri.org billion in funding as of December 2016. ${ }^{1}$ Despite the growth of PER, there are skeptics and real challenges to broadening research teams to include patients and all other stakeholders. Bombak and Hanson noted the dangers related to the "lack of critical scholarship and wholesale investment into narrowly defined methods of conducting patient engagement." "2 For example, they cited the risk of marginalizing patients not participating in engagement activities, the potential for "tokenism," and inadequate measures and reporting. Hahn et al also discussed tokenism, specifically that it is less about the 
structure of the patient-stakeholder engagement and instead is "all about the intent" and that each aspect of engagement may be measured on a scale from "token" to "genuine."3

Bombak and Hanson called on researchers to recognize the value of qualitative and community-based participatory research, which has a long track record of involving patients as partners at varying levels. ${ }^{2}$ It is only with rigorous evidence about the impact of patient engagement that the community can make strides toward exploring the best recipes for engagement (eg, who, when and how much).

While PER is increasingly valued and more routinely implemented in practice, the measurement of the impact of this engagement on research outcomes is underdeveloped. ${ }^{4}$ The importance of PER was recognized by the U.S. Patient Protection and Affordable Care Act of 2010, which led to the creation of PCORI, the first funding agency that deliberately supports research that is "guided by patients, caregivers, and the broader healthcare community." 5 PCORI and others have made progress in identifying outcomes, measures and methods to assess impact on the engagement process and on those involved. ${ }^{4,6-8}$ Although there are anecdotal indications that engaging patients leads to improved study design and research methods, there is limited systematic evidence to demonstrate this impact. And while there are many examples of innovative attempts to measure the impact of patient engagement in public discourse and community-based participatory research, ${ }^{9}$ researchers need measurement tools and principles that apply to all research studies (randomized controlled trials, observational studies, qualitative studies, data-only studies, etc.) that engage patients as research team members and advisors in order to conceptualize, design and conduct research and disseminate findings relevant to patient needs and concerns.

The PCORI-funded Critical Outcomes of Research Engagement (CORE) workshop described herein was developed to follow up on work initiated at a PER symposium held in conjunction with the 2016 Health Care Systems Research Network (HCSRN) Conference. Discussion at that symposium and the subsequent article on methods for engaging patient partners emphasized the critical importance of measuring the impact of PER and called for further methods development in this area. ${ }^{10}$ Prior to the CORE workshop, the planning team searched the literature for existing assessment questions and measures that were relevant to the study of the impact of PER. While some tools exist for measuring and evaluating the process of PER - the GRIPP checklist, ${ }^{9}$ the PCORI WE-ENACT surveys, ${ }^{11}$ and the Wilder Collaboration Factors Inventory ${ }^{12}$ - less attention has been paid to measuring the impact on research outcomes across the continuum, from developing research questions to disseminating final results. ${ }^{4,13}$

A report by Lavallee et al and a presentation by Hamilton and True identified process constructs as including "what happens in engagement and how." $6,10,14$ Meanwhile, impact constructs include what is changed as a result of the engagement and how big that change is. For example, including patient partners in developing a questionnaire is a process change and a better response rate on the questionnaire would be an impact change. Esmail et al describe that impact evaluation - which evaluates the "intended effects of engagement" — includes quality and validity of results, improved recruitment, changes in health outcomes, etc. ${ }^{4}$ Another important outcome of PER relates to the ethics of research and research procedures, for example, improving informed consent processes ${ }^{15}$ and addressing health inequities, conditions and issues that can lead to improvements in community health. ${ }^{16}$

PCORI's conceptual model for patient-centered outcomes research ${ }^{17}$ provides additional targets for moving toward measuring the impact of patientengagement efforts. This connected longitudinal framework reflects broad areas for impact evaluation, from the foundational elements that can provide a receptive environment for engagement, through effective actions in the engagement process itself, to desired near-, intermediate- and long-term outcomes that include creating effective partnerships, conducting research activities that are patient-centered, generating results meaningful to stakeholders, and improving health. ${ }^{18}$

We describe how a planning committee of patient partners and researchers from HCSRN member sites - Kaiser Permanente Colorado Institute for 
Health Research (Denver, CO), Marshfield Clinic Research Institute (Marshfield, WI), Palo Alto Medical Foundation Research Institute (Palo Alto, CA), Henry Ford Health System Department of Public Health Sciences (Detroit, MI) and Kaiser Permanente Washington Health Research Institute (Seattle, WA) collaboratively coordinated a workshop and follow-up activities aimed at: 1) identifying the desired outcomes of patient-engaged research at each step in the research process; 2) identifying how patient engagement could influence the research process and its outcomes; and 3) developing a synthesis and distillation of CORE components that capture the potential impacts of patient engagement on research.

\section{Review of Existing Constructs, Instruments}

Some existing instruments skew toward measuring the process of PER more than its impact on research outcomes. WE-ENACT specifically addresses the role and responsibilities of patients and other stakeholders in PCORI-funded projects with surveys completed by researchers, patients and stakeholders annually. ${ }^{17}$ The surveys cover measures related to the process of engagement as well as self-reported outcomes. Potential limitations of WE-ENACT include infrequent data collection, response bias and the inherent conflicts when a funder collects survey data on a funded project.

The GRIPP checklist provides a comprehensive plan for standardizing the reporting of patient and public involvement when writing about engagement. ${ }^{9}$ However, the checklist includes over 30 conceptual items that require extensive data collection resources and increased time devoted to analysis. It also anticipates ample space in manuscripts. While this may be feasible as a model for comprehensive reporting in engagement-focused manuscripts, space limitations may make it difficult to integrate this level of detail on PER methods and impact into manuscripts addressing primary research findings. Importantly, the GRIPP checklist does not support systematic collection of quantified discrete elements.

Brett and colleagues' systematic review on the impact of public and stakeholder involvement on health research highlighted outcomes and other important components that should be measured but are rarely addressed. ${ }^{13}$ These include engagement in the pre-proposal, proposal writing and other phases of research; challenges experienced; and how engagement affects time, workloads and costs. ${ }^{13}$ The authors recognized the need for both qualitative and quantitative measures as well as better descriptions of how, where and when engagement was conducted in order to improve our understanding of its potential beneficial impacts.

Lavallee et al list six meta-criteria, or ideal attributes of patient engagement, for comparative effectiveness research: respect, trust, legitimacy, fairness, competence, and accountability. ${ }^{6}$ Potential measures, which generally focused on the process of engagement and impact on the people involved, also were suggested. A similar approach is the Wilder Collaboration Factors Inventory, a brief questionnaire measuring 20 factors related to the collaboration process. ${ }^{12}$ These constructs and instruments are important to the PER field; however, they are mainly focused on the impact on the people involved in the research partnership rather than the short- and long-term outcomes of collaboration.

As noted, the existing measures and approaches to evaluating the impact of patient engagement on the conduct of research, and particularly on research outcomes, are limited. Therefore, the CORE workshop was designed to focus on this gap in the science of patient-engaged research.

\section{METHODS}

The workshop was held in Denver, Colorado, October 26-28, 2016, and included 5 patient partners, who also served on the workshop planning committee, and 24 researchers from academic and research organizations, health care systems, federal and community-based organizations, and representatives from PCORI. The researchers on the planning committee were members of HCSRN's Patient Engagement in Research Scientific Interest Group. The 5 patient partners were female, racially/ethnically diverse, came from different areas of the country, and had extensive personal and professional experience relevant to health care but varying levels of experience with research. Three were very experienced in research and quality improvement projects with health systems, while the other two were new to research. Patient partners were recruited by a combination of outreach to patients already active in PER and by reaching out to patients who had volunteered with their local health systems' patient 
advisory programs. Each patient partner worked in tandem with her recruiting researcher to represent their site and to ensure the partner was comfortable in her role and knew what was to be expected.

The planning committee met by phone and webinar at least two times per month for three months prior to the workshop, and used a document-sharing website for communications and developing materials. Researchers and patient partners collaboratively set the agenda and tone for the workshop, the goal of which was to identify core principles and relevant outcomes that could support a systematic approach to measuring the impact of patient engagement on the conduct and outcomes of health research. Importantly, the workshop aimed to embody the principles of engagement. Patient partners provided strong voices and decades of experiences as patients and community activists. Most of the dynamic workshop agenda was devoted to a combination of small group and interactive full group facilitated sessions.

The first task was to generate ideas on desired outcomes of research through dialogue and clustering exercises. Participants worked at tables in small groups, with a patient partner present in each. Each small group was responsible for initial discussion within one or two of the eight stages of research (Table 1). Each group noted emerging themes and categorized these common constructs for each research stage onto large poster boards. Collectively, the large group further synthesized these emerging common constructs into what were considered to be the most essential, or "CORE," components. Following these steps, a discussion was conducted to determine how these COREs would be measured.

\section{Table 1. Research Stages}

1. Develop the question

2. Develop the proposal/study design

3. Administrative

4. Recruitment

5. Conduct research/data collection

6. Data analysis

7. Write-up \& dissemination

8. Implementation

\section{RESULTS}

\section{CORE Components}

For each stage of research, the workshop participants iteratively shortened the long list of suggested outcomes into a briefer list of common constructs, then selected a smaller subset deemed the most critical (ie, COREs). While there were some CORE components unique to specific stages of research, there was a group of COREs that had universal relevance across all phases of PER, which were identified as "overarching principles." Other components considered desirable in all research whether or not patient partners were engaged were labeled "Research 101."

Table 2 lists and defines the CORE components identified by workshop participants. The shared discussion kept returning to these themes as being the most important goals of PER, including the concept of patient-centeredness, being meaningful and relevant to patients and communities, collaborative efforts, and use of a common language to make the work more understandable. Values like respect, transparency, timeliness and integrity also surfaced repeatedly during the group discussions, suggesting an elaboration of patient-centeredness that reflects a moral perspective on the process of establishing and maintaining the relationships required to conduct meaningful patientengaged research.

Table 2 also summarizes concrete outcomes that would be associated with these related measures. Relevant questions that could assess attainment of these COREs were identified. For example, possible measures for team collaboration included: "What is the patient partner's comfort level 1) during discussions, or 2) with written materials?" For rigorous, a checklist to capture at which stage patient partners were consulted and had a meaningful influence was proposed. A suggested measure for the ethical and transparent CORE was: "How does the study design, including data collection methods, accommodate and show respect for participant diversity?" These suggested measures served as a starting point for developing a coherent assessment framework that could help researchers identify desired outcomes of patient-centered research, as well as measure and connect the impacts of those on the research process and results (Figure 1). 
Table 2. Critical Outcomes of Research Engagement (COREs) Definitions, Related Measures

\begin{tabular}{l}
\hline CORE \\
\hline Patient-centered - Research centered around \\
patients' values, beliefs and experiences. \\
Anticipates participant issues, respects and \\
reflects patient experience and validates patient \\
partner input/contributions. Engages patients in \\
a respectful, culturally appropriate and condition/ \\
disease-sensitive manner.
\end{tabular}

Meaningful - Research/methods/outcomes are reflective of and relevant/meaningful to the community as well as impactful (as perceived by community and all stakeholders).

Team collaboration ("teamness") - Having an appropriate, collaborative team that includes a variety of researcher and patient expertise and experiences. Capacity building includes patient partners gaining skills and knowledge through experience and/or training.
Measures

1. How did patient partners influence each stage of research and critical research tasks?

2. Changes in specific outcomes (response rates, retention, etc.) relative to other similar studies without patient partners.

1. Are potential outcomes, comparators and content meaningful to patient community?

1. What is the patient partner's comfort level during discussions?

2. What is the patient partner's comfort level with written materials?

3. Do all team members trust and respect each other?

Understandable - Use of engaging common
language that includes specific and measurable
questions, agreed on by both researchers
and partners. All research materials are
understandable to participants and patient
partners.

Rigorous - Research is generalizable, reliable and validated by the patient partners and reflects the diverse participants in an ethical, unbiased and timely fashion. Honest/accurate results.
1. Are study materials understandable and written in a common/plain language?

2. Evaluate the reading level of research documents.

3. How were patient partners trained and supported in their research work?

4. Did researchers present data to patient partners in an accessible, understandable way?

1. Did the research team use "realistic continuous improvement" methods to maintain scientific rigor while also incorporating partner suggestions?

2. Checklist of stages/discrete decisions for which patient partners were consulted and had meaningful influence.

3. Did patient partners propose any changes that were not made? If so, explain why.

Integrity/Adaptable - Research maintains balance between process improvement and study goals. Willingness to change study design through "realistic continuous improvement."

1. Is the research question clear and understood by everyone?

2. To what extent do patient partners contribute to creating a fair, ethically sound research study (document changes in study design, methods, materials, etc.)?

Legitimate - Findings are considered legitimate and trusted by relevant communities, increasing likelihood results will be translated/adopted due to buy-in.

1. To what extent were partners involved in each stage of research? How were their insights incorporated?

2. To what degree was the sample or study population diverse and representative/unbiased?

Feasible - Identify/address assumptions to make goals and methods realistic.

1. Are research goals and methods realistic and feasible?

Ethical and transparent - Patient partners ensure transparency, fairness, truly informed consent and participation, and continuously check assumptions of research team members.

Timely - Timely analysis and reporting; iterative data sharing with patient partners.

Sustainable - Research has long-term value; patient partner relationships are maintained over time.
1. Are all methods and materials patient-friendly?

2. How does the study design, including data collection methods, accommodate and show respect for participant diversity?

3. Is data/privacy protection more patient-centered and/or changed?

1. Is conduct of research and sharing information with patient partners timely?

1. What different mediums were used to disseminate findings, and where were results shared?

2. Were results translated or adopted outside the research study?

3. What role did patient partners play in dissemination? 


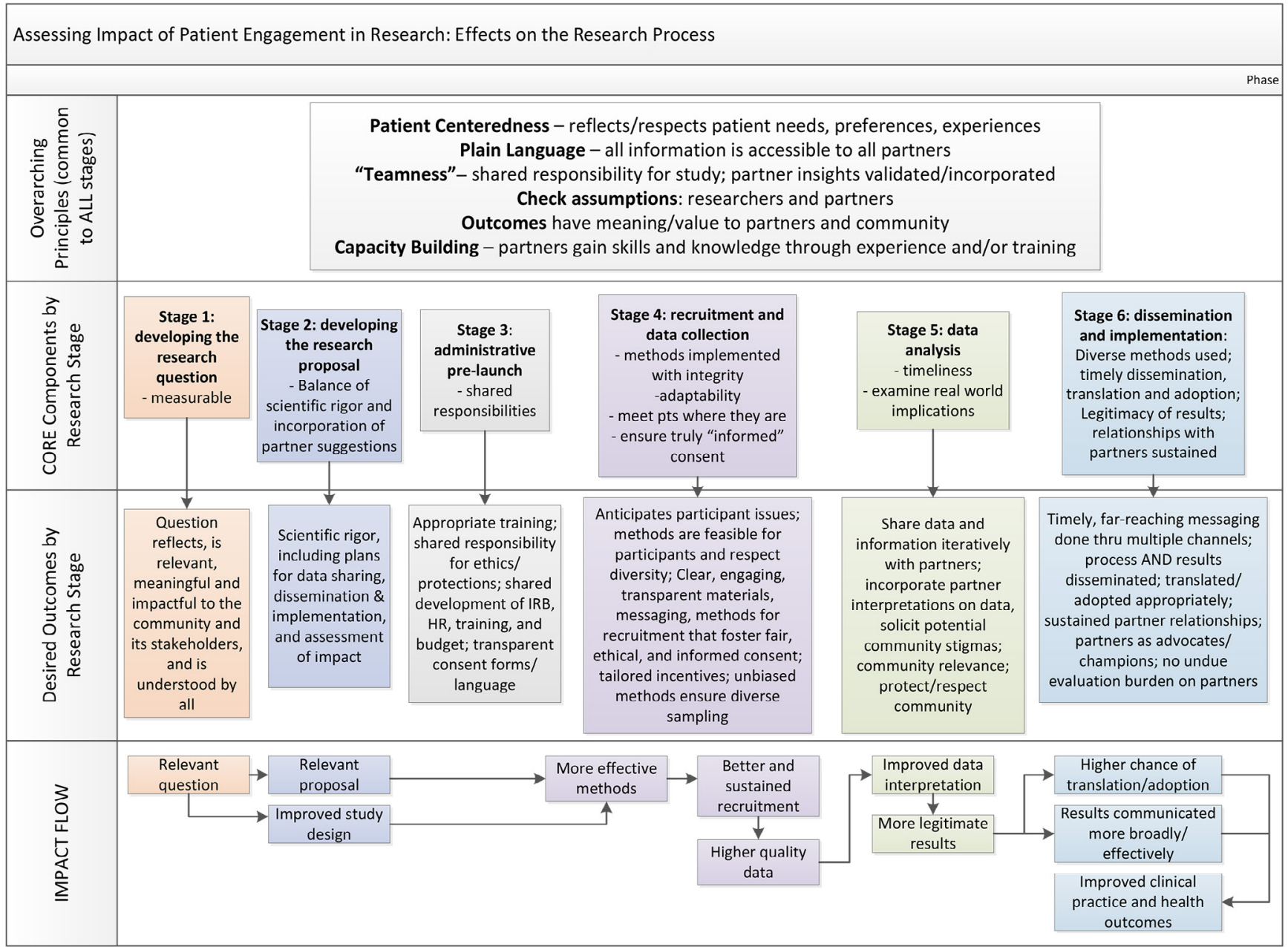

Figure 1. Flow diagram of hypothesized patient engagement in research outcomes.

Impact measures associated with critical downstream outcomes of the quality and influence of research results, and the corresponding health of patients and populations, were envisioned at the workshop and are reflected in initial form within some of the COREs. For example, response rates and subject retention are proposed measures affiliated with the patient-centered CORE, and the meaningful CORE anticipates study outcomes that are relevant to patient communities. Figure 1 illustrates how patient-centered strategies may have positive impacts that flow from each stage of research to the next and finally result in improved health outcomes for individuals and communities. For example, a more relevant and meaningful research question developed in partnership with patients may lead to a more relevant proposal and improved study design, more effective methods, better participant recruitment and higher quality data, improved data interpretation and more legitimate results, and higher likelihood of translation and adoption of research results into everyday practice, as well as broader and more effective communication of findings. All of this, in turn, should lead to improvements in clinical practice and health outcomes.

\section{Proposed Methods for Measurement}

In addition to making progress toward identifying the most critical process and outcome constructs to measure, workshop participants discussed who would collect the relevant data as well as how, when and how often. For example, paper questionnaires, online questionnaires, interviews, journaling, checklists and retrieving data from study documents are all potentially viable approaches to initial assessment. The participants discussed who should collect this information and the potential for bias when having either the research team or the funder collect the data. The timing of when to collect data, both timing relative to study milestones 
and frequency of data collection, was discussed to strike a balance between collecting meaningful data without overburdening respondents with work.

However, participants also agreed that the work required to collect data on the impact of patient engagement is a challenge that requires significant resources and significant time investment. Since all participants agreed that doing this meta-level of research was necessary to define the value of patient engagement, it was suggested that easy-to-complete checklists or report card-like instruments, modeled after the Wilder Collaboration Factors Inventory, may be appropriate. Participants suggested that the research community decide on a base or default set of measures and then supplement these with add-on modules or data-collection methods to capture the nuances and diversity in study designs and methods. Workshop attendees noted that each project may need to revisit and refine some measures based on its study design, research team and scope.

As noted in a report by Lavallee et al, "Ideally, an evaluation should be given at a time that both maximizes memory and leaves adequate time for reflection. Also, since engagement should be a continuous process, it may be beneficial to conduct a series of evaluations over the lifetime of a project, allowing opportunities to adjust activities in response to feedback." "We agree that a repeated series of assessments allows for sharper identification of connection points between engagement effects in early phases of research and impacts in later phases. For a higher-level conceptualization, Figure 1 illustrates the CORE components generated by stage in the workshop discussion, elaboration on corresponding outcomes for assessment, and a connected series of stage-specific impacts that show how the effects of research engagement may flow from early work to form patient-centered research questions all the way downstream to more legitimate research results and greater adoption.

\section{DISCUSSION}

Building on PCORI's conceptual framework of patient-centered outcomes research, this workshop generated a distilled list of critical outcomes of research engagement by research stage and mapped these outcomes to existing and potential assessment questions. Mapping outcomes of interest to existing measures ${ }^{6,9,11,17}$ and constructing potential measures revealed an abundance of instruments that capture the processes of engagement and fewer that evaluate the influence of PER on research projects and their outcomes.

The evaluation found most synergy between COREs and meta-criteria suggested by Lavallee et al, such as respect, legitimacy and accountability, ${ }^{6}$ and items from the WE-ENACT survey, such as: "For each part of the research project, describe what you did and how it made a difference." ${ }^{11}$ Measures addressing the early stages of research were more abundant, whereas measures to evaluate the impact of PER on later stages, like data analysis and dissemination/implementation of findings, were scarce. Over time more attention will likely be paid to these and other longer-term outcomes of PER, such as the critical question of the impact on health and health care, as envisioned in PCORI's conceptual framework. ${ }^{18}$ More nuanced questions that have been raised - including documentation of unintended consequences of engagement, how best to mitigate them, and where/when in the research process engagement offers the greatest yield ${ }^{2,8}$ — hopefully can be addressed through sustained evaluation.

While work remains to generate and pilot efficiently measureable concepts for robust routine evaluation, we believe that both this workshop and its products to date are instructional to the research engagement community and facilitate necessary movement toward the long-term goal of assessing engagement's impact on health.

The field of patient-engaged research continues to examine what it means to have authentic and effective engagement partnerships, with extensive consideration of the roles of researchers and partners at each stage of research. ${ }^{19}$ Accordingly, workshop discussions and the products of this enterprise focused on engagement outcomes by research stage, which allowed both for a more granular consideration of engagement impact and establishment of a framework for a serial approach to assessment that could maintain the natural connectedness of stage-specific impacts as the work progresses. While practicality is a pending question, there is common agreement on the importance of ongoing assessment and feedback. ${ }^{1}$ 


\section{Strengths}

The proposed measures recognized and addressed the practical limitations inherent in any standard assessment. Due to resource constraints, researchers cannot, and likely should not, involve patients in every discussion and every decision at every step of the way. Likewise in engagement evaluation, measurement at every step would not be feasible. However, the aim should be to provide useful patient engagement outcomes to construct a tool that can balance scientific robustness with feasibility and ease of use. It has been suggested by some that extensive qualitative assessment would provide the most informative evaluation, ${ }^{20}$ but that is not scalable for the systematic collection of discrete quantified elements that would be needed to build the evidence base, compare impact across projects or evaluate changes over time.

\section{Limitations}

We encountered several challenges in organizing and conducting this workshop. First, despite providing definitions in background materials and introductory presentations, participants struggled with the semantics of several terms. For example, terms such as "impact," "outcome," and "construct" held different meanings across workshop attendees. Collectively, there also was periodic difficulty disentangling focus on outcomes that reflected improvement in the process of engagement from the outcomes of engagement on research projects and their results. While participants self-monitored and checked and adjusted during workshop sessions to limit this, group discussion tended to return to a blending of how patient engagement could impact the engagement process along with how it could impact the research process and its outcomes.

Also, whereas the group seemed to find it most easy to discuss the importance of trust, respect and inclusiveness for the people involved, and was able to propose general outcomes of interest by research stage, there was more difficulty articulating with the desired specificity measurable ways engagement would change or improve the conduct of the research, such as the quality of data or interpretation of results. Orienting patient partners in relevant research terms and methodologies is an essential component for meaningful patient participation and maximizing engagement value to create action. ${ }^{21}$ While the patient partner may not be a content expert or familiar with the terms or methods, using common language and orienting everyone to the concepts gives the patient the tools to be able to contribute during all stages of project development and reinforces the idea that they are research partners, not research subjects. Additionally, patient partners provide invaluable feedback on feasibility from the research subject's perspective, making the study more patient-friendly and focused. Measurements can be taken of the number of patient partner-induced changes in research methodologies that improve data collection, relevancy and target population validity and credibility. Finally, participants recognized that directly measuring impacts of engagement on health outcomes remains a challenge for the broader scientific engagement community to tackle as the growing portfolio of engaged research projects matures.

Because the group was diverse in terms of geography, research experience and point of reference, some struggles were addressed both during the workshop and on the organizing committee to ensure there was mutual understanding of our goals, our roles, and how to participate and contribute in a meaningful way. Our patient partners alerted us when research jargon crept into our language, and this afforded the opportunity to examine again the importance of using common language. Also, due to a rapid project timeline, there was little time to devote to patient-partner training. While this did not present a significant challenge, the committee took the extra time, when needed, to revisit some of the fundamental topics that would have been covered in more traditional patient-partner training.

Throughout the project the researchers on the planning committee were cognizant of the comfort level, knowledge, experience and perspective of the patient partners. By checking in frequently with the patient partners, and offering one-on-one conversations and refreshers in certain research terminology and workflows, all members of the planning committee benefited from increased clarity and understanding. The planning team often returned to discussing the feedback loop and gray area between research outcomes and engagement outcomes. Patient-partner participation in developing a research question is both an engagement outcome and a research outcome when it leads to more meaningful questions being asked. While these outcomes are conceptually distinct, in reality there is often overlap and linkage; better participant recruitment may be both a research and engagement outcome. 


\section{Implications for Future Research}

Through closing discussions, the workshop participants agreed that next steps would include: 1) generating written summaries of the workshop and its products to be disseminated to relevant audiences; 2) mapping the COREs to reportable measures by identifying or creating useful metrics to capture data on these components, placing these measures along the PCORI evaluation framework, and identifying and filling gaps as needed; 3) building a community of practice through continued engagement with workshop attendees, plus casting a wider net to identify other individuals, communities (eg, INVOLVE ${ }^{22}$ ) and instruments that can be leveraged in future work; and, 4) piloting CORE measures, and obtaining buy-in from others in the community of practice to pilot these measures in their own work.

There is much to learn from understanding how participants (patients, researchers, other stakeholders) affect the process of conceptualizing, designing, implementing and completing a research project. Discovering how the research process evolves differently with the addition of patient partners to the research team could provide clues for identifying longer-term effects of patient-engaged research.

\section{CONCLUSIONS}

Patient engagement is not conducted the same way for every research project. In order for us to maximize its effectiveness and illustrate its value, we must determine in what ways and at which stages of research patient partners affect the research process and enhance the impact of research. Once pinpointed, we may be able to link that engagement to more relevant research results. Theoretically those results could be more easily translated to and adopted for clinical practice and may then lead to improved health outcomes for patients.

This workgroup of researchers and patient partners was able to create a list of questions that could address the gap in instruments available for measuring the impacts of engagement on research itself. The products of the workshop were a flow chart of ways patient partners could contribute to the research process and a list of topics and questions to ask to determine whether changes occurred. Future research is needed to create a valid and reliable survey, as well as to validate whether these topics and questions improve our understanding of how having patient partners as members of a study team changes the research process.

As the executive director of PCORI, Joe Selby, has noted, "[PCORI] will be evaluated ultimately on whether the research we fund can change clinical practice and help reduce the variations and disparities that stand between patients and better outcomes." 23 It is only through careful measurement of the impact of patient engagement in research that we can truly identify whether it has ultimately benefited individuals' health or improved our health care system.

\section{Patient-Friendly Recap}

- Researchers and funding agencies use surveys that ask patient partners about their role, responsibilities and satisfaction as members of study teams. But these surveys rarely ask how patients, as team members, truly affect the course of the health research.

- A workshop made up of researchers and patient partners evaluated how to measure the impact of patients engaged in the research process, with researchers sharing their professional experiences and patients contributing their experiences as those who have received care.

- Products of the workshop (which, once validated, could be incorporated into national frameworks) included 1) a flow chart of ways patient partners can change the research process, and 2) a list of topics and questions to ask to measure whether change happened.

\section{Acknowledgments}

We are grateful to the Patient-Centered Outcomes Research Institute (Washington, DC) for project funding; to Lia Hotchkiss, director of the Eugene Washington PCORI Engagement Award Program, and Lauren Massey, PCORI Contracts Operations, for their guidance and support; to the Daniels Fund (Denver, CO) for providing a meeting space for the workshop; to Dr. Laura Forsythe of PCORI and Dr. Danielle Lavallee of the University of Washington (Seattle, WA) for their keynote presentations and 
active participation in the workshop; and to all workshop attendees for their enthusiastic engagement. Many thanks to members of the workshop planning committee: Gina Napolitano, Ursula Salas, Heather Tabano, Deb Multerer, Mary Schramke, Jacquetta Hinton and Neely Williams. We also are grateful to the Health Care Systems Research Network and the HCSRN Patient Engagement in Research Scientific Interest Group for facilitating collaboration. Lastly, we are indebted to the health care systems that supported this patient-engagement work: Kaiser Permanente Colorado, Marshfield Clinic Health System, Palo Alto Medical Foundation, Henry Ford Health System, and Kaiser Permanente Washington.

\section{Conflicts of Interest}

None.

\section{Funding Sources}

Research reported in this article was funded through a Patient-Centered Outcomes Research Institute Award (EAIN-3398; PI: Greenlee). The statements in this publication are solely the responsibility of the authors and do not necessarily represent the views of PCORI, its Board of Governors or Methodology Committee.

\section{References}

1. Patient-Centered Outcomes Research Institute. Research funding. http://www.pcori.org/sites/default/files/PCORIResearch-Funding.pdf. Accessed January 20, 2017.

2. Bombak AE, Hanson HM. A critical discussion of patient engagement in research. J Patient Cent Res Rev. 2017;4:39-41. CrossRef

3. Hahn DL, Hoffmann AE, Felzien M, LeMaster JW, Xu J, Fagnan LJ. Tokenism in patient engagement. Fam Pract. 2017;34:290-5. CrossRef

4. Esmail L, Moore E, Rein A. Evaluating patient and stakeholder engagement in research: moving from theory to practice. J Comp Eff Res. 2015;4:133-45. CrossRef

5. Selby JV, Beal AC, Frank L. The Patient-Centered Outcomes Research Institute (PCORI) national priorities for research and initial research agenda. JAMA. 2012;307:1583-4. CrossRef

6. Lavallee DC, Williams CJ, Tambor ES, Deverka PA. Stakeholder engagement in comparative effectiveness research: how will we measure success? J Comp Eff Res. 2012;1:397-407. CrossRef

7. Deverka PA, Lavallee DC, Desai PJ, et al. Stakeholder participation in comparative effectiveness research: defining a framework for effective engagement. J Comp Eff Res. 2012;1:181-94. CrossRef

8. Forsythe LP, Ellis LE, Edmundson L, et al. Patient and stakeholder engagement in the PCORI Pilot Projects: description and lessons learned. $J$ Gen Intern Med. 2016;31:13-21. CrossRef
9. Staniszewska S, Brett J, Mockford C, Barber R. The GRIPP checklist: strengthening the quality of patient and public involvement reporting in research. Int $J$ Technol Assess Health Care. 2011;27:391-9. CrossRef

10. Madrid S, Tuzzio L, Stults CD, et al. Sharing experiences and expertise: the Health Care Systems Research Network workshop on patient engagement in research. J Patient Cent Res Rev. 2016;3:159-66. CrossRef

11. Patient-Centered Outcomes Research Institute. Ways of Engaging- ENgagement ACtivity Tool (WE-ENACT) patients and stakeholders 2.0 item pool. Posted December 16, 2014. http://www.pcori.org/sites/default/files/PCORIWE-ENACT-Patient-Stakeholder-Survey-Item-Pool.pdf. Accessed March 9, 2017.

12. Mattessich PW, Murray-Close M, Monsey BR. The Wilder Collaboration Factors Inventory: Assessing Your Collaboration's Strengths and Weaknesses. Nashville, TN: Turner Publishing Company, 2001.

13. Brett J, Staniszewska S, Mockford C, et al. Mapping the impact of patient and public involvement on health and social care research: a systematic review. Health Expect. 2014;17:637-50. CrossRef

14. Hamilton A, True G. Measuring processes and impacts of veteran-engaged research. Presented at the Veteran Engagement Initiative in VA Research Cyberseminar (May 17, 2016). https:// www.hsrd.research.va.gov/for_researchers/cyber_seminars/ archives/1126-notes.pdf. Accessed April 4, 2017.

15. National Health Service's Health Research Authority, INVOLVE. Impact of public involvement on the ethical aspects of research. Published May 2016. http://www.invo. org.uk/posttypepublication/impact-of-public-involvementon-the-ethical-aspects-of-research/. Accessed April 4, 2017.

16. Woolf SH, Zimmerman E, Haley A, Krist AH. Authentic engagement of patients and communities can transform research, practice, and policy. Health Aff (Millwood). 2016;35:590-4. CrossRef

17. Patient-Centered Outcomes Research Institute. PCORI Evaluation Framework 2.0. Published August 11, 2015. https://www.pcori.org/sites/default/files/PCORI-EvaluationFramework-2.0-Executive-Summary.pdf. Accessed August 8, 2017.

18. Frank L, Forsythe L, Ellis L, et al. Conceptual and practical foundations of patient engagement in research at the patient-centered outcomes research institute. Qual Life Res. 2015;24:1033-41. CrossRef

19. Fagan MB, Morrison CR, Wong C, Carnie MB, GabbaiSaldate P. Implementing a pragmatic framework for authentic patient-researcher partnerships in clinical research. J Comp Eff Res. 2016;5:297-308. CrossRef

20. Staniszewska S, Adebajo A, Barber R, et al. Developing the evidence base of patient and public involvement in health and social care research: the case for measuring impact. Int $J$ Consum Stud. 2011;35:628-32. CrossRef

21. Anderson M, McCleary KK. On the path to a science of patient input. Sci Transl Med. 2016;8(336):336ps311. CrossRef

22. National Health Service. INVOLVE home page. http://www. invo.org.uk/. Accessed January 20, 2017.

23. Selby J. PCORI's research will answer patients' real-world questions. Posted March 25, 2014. http://healthaffairs.org/ blog/2014/03/25/pcoris-research-will-answer-patients-realworld-questions/. Accessed April 4, 2017.

(C) 2017 Aurora Health Care, Inc. 